\title{
Propuesta metodológica
}

para la implementación

y el seguimiento

de un plan de estudios

Comité de Seguimiento e Implementación del Plan

de Estudios de la Licenciatura en Arquitectura $2012^{1}$

on base en las recomendaciones de la Acreditadora Nacional de Programas de Arquitectura y Disciplinas del Espacio Habitable A.C. (ANPADEH) y con el objeto de verificar que los programas de las asignaturas cumplan con los requisitos de estructura curricular exigidos, revisar los objetivos y contenidos de los cursos, así como precisar la orientación académica, ideológica y profesional de la estructura curricular, el Centro de Ciencias del Diseño y de la Construcción conformó el Comité para el Seguimiento y la Implementación del Plan de Estudios de la Licenciatura en Arquitectura 2012. ${ }^{2}$

Este comité elaboró un Plan Estratégico donde se da prioridad a la revisión del nivel de conocimiento de acuerdo a los objetivos de aprendizaje y su congruencia con los contenidos en el diseño de los programas de las asignaturas, y establece el guión metodológico para tal fin, fundamentado en el Modelo Educativo Institucional $(\mathrm{MEI})^{3}$ y en la metodología establecida por la Dirección General de Docencia de Pregrado de la UAA para la revisión y diseño de planes de estudio y de los programas de materias.

\section{Definiciones conceptuales y base metodológica}

El diseño del plan de estudios de la Licenciatura en Arquitectura se apega al MEI, por lo que las bases teórico metodológicas establecidas por el comité para el seguimiento e implementación del plan de estudios se derivan de sus postulados en cuanto los procesos y experiencias de aprendizaje y enseñanza, ${ }^{4}$ que de manera selectiva y resumida se anotan a continuación.

\section{Proceso de aprendizaje}

- El aprendizaje es la finalidad de la enseñanza. ${ }^{5}$

- Se aspira a un aprendizaje significativo de calidad, integral, y centrado en el estudiante que coadyuve a la consecución de los fines del MEI.

- El contenido del aprendizaje es el conjunto de conocimientos, habilidades, actitudes y creencias que el estudiante deberá poseer.

- El aprendizaje debe ser cimentado en los conocimientos previos de los estudiantes.

- El aprendizaje tiene como uno de sus propósitos la solución de problemas reales del entorno natural y social, logrando así un aprendizaje significativo.

1 Mario Eduardo Zermeño de León, Blanca Ruiz Esparza Díaz de León, Alberto Sánchez López, Juan Jesús Aranda Villalobos, José Díaz Ríos, Carlos Parga Ramírez, Antonio Rosales Hernández, Aarón Ruiz Esparza Gutiérrez, Ernesto Tello Ruiz, Alejandra Torres Landa López y Humberto Vázquez Ramírez.

2 Autorizado por el H. Consejo Universitario en septiembre del año 2011.

3 Aprobado por el H. Consejo Universitario en sesión celebrada el 15 de diciembre del año 2006.

\footnotetext{
4 Se trata de dos de los componentes del hecho educativo, a través de los cuales se busca concretar las aspiraciones que en materia de formación se han planteado en el MEl. Cabe resaltar que se indica primero el proceso de aprendizaje para hacer énfasis en la posición central que éste debe tener en el proceso educativo.
}

5 El mel es un modelo centrado en el aprendizaje del alumno. 


\section{Proceso de enseñanza}

- La enseñanza es una actividad fundamental en el proceso educativo. Se trata de un proceso de intervención, creativo, intencionado y sistemático, con objetivos explícitos de facilitar la interacción del estudiante con los contenidos, a fin de construir nuevo conocimiento.

\section{Experiencias de aprendizaje}

Las experiencias de aprendizaje son el resultado de la interacción del estudiante con el entorno de aprendizaje (escenarios, contenidos, actores del proceso educativo, formas de organización y de trabajo, instrumentos y tareas de cada una de las actividades), con la finalidad de desarrollar habilidades como el razonamiento, el análisis de la información, la reflexión, la expresión oral y escrita y la manifestación de actitudes y valores que promueve la institución.

Estos postulados deben estar contenidos en los programas de las materias por impartir en la licenciatura, y por ello es uno de los principales cometidos del comité, y se apega a lo establecido que considera al aprendizaje como: el conjunto de conocimientos, habilidades, actitudes y creencias que el estudiante deberá poseer.

El conocimiento ${ }^{6}$ como requisito inicial del conjunto se considera que es un proceso de adquisición de las dimensiones del "aprendizaje" (Pérez, 2006) (ver Imagen 1).

Estas dimensiones se clasifican por categorías taxonómicas (Anderson et al., 2001) y a su vez se agrupan por nivel (ver imágenes 2 y 3 ).

Por su parte, las "habilidades" son entendidas como concepto genérico susceptible de muchas acepciones particulares, clasificadas por sinónimos; se concretan en los siguientes grupos: habilidad corporal y manual (versus destreza); habilidad intelectual (versus, ingenio); habilidad en el trato social (versus, tacto).

6 El conocimiento incluye aquellos comportamientos y situaciones de examen que acentúan la importancia del recuerdo de ideas, materiales o fenómenos, ya sea como reconocimiento o como evocación. La conducta que se espera de un estudiante en situación de evocación es similar a la que se esperó de él durante el aprendizaje original, cuando se deseó que almacenara en su mente determinada información. Posteriormente, se le pide que recuerde esa misma información (Bloom, 1971).

7 El que sabe hacer una cosa bien y con conocimiento de lo que hace, tiene habilidad; el que la hace materialmente bien y con facilidad, tiene destreza. Aquélla se refiere directamente al saber; ésta se refiere directamente al ejecutar. Un artífice tiene habilidad cuando sabe ejecutar bien la obra que le encargan;y destreza en el manejo material de los instrumentos de su profesión. Un maestro tiene habilidad para enseñar cuando sabe el buen método y los medios que debe emplear para ello.

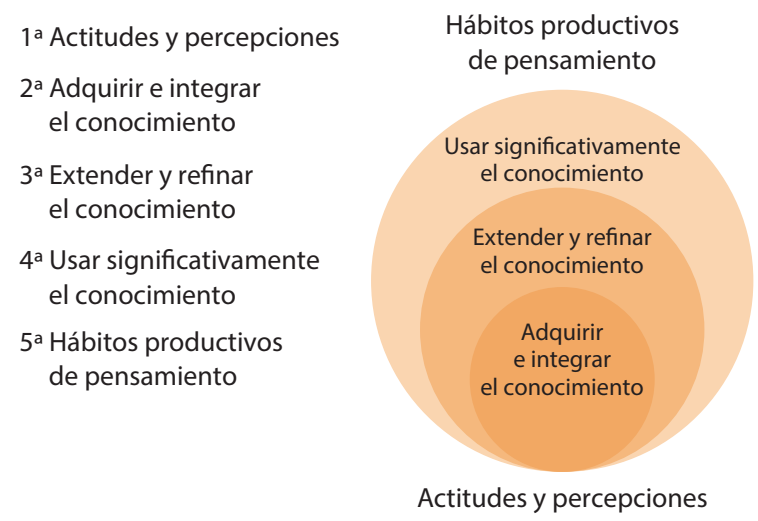

Imagen 1. Dimensiones del aprendizaje.

Fuente: Pérez, 2006.

La taxonomía de Bloom revisada por Anderson

Niveles originales de Bloom

- Conocimiento

- Comprensión

- Aplicación

- Análisis

- Síntesis

- Evaluación

Imagen 2. Categorías taxonómicas.

Fuente: Anderson et al., 2001.

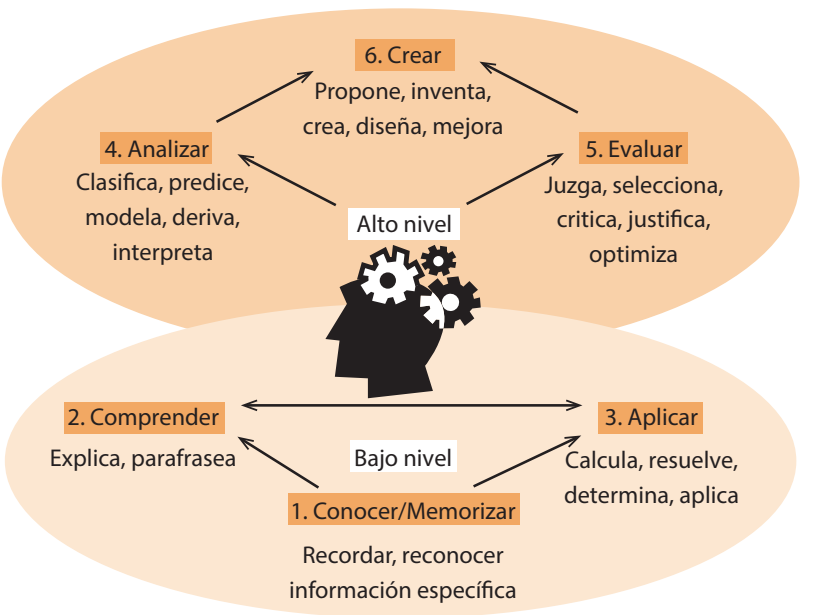

Imagen 3. Niveles taxonómicos.

Fuente: Pérez, 2006.
Niveles revisados por Anderson (2001)

- Memorizar

- Comprender

- Aplicar

- Analizar

- Evaluar

- Crear 
Para esta comisión, el concepto de "habilidades" es definido como la capacidad en el conocimiento o "habilidades cognitivas", ${ }_{8}$ y son definidas a través de operaciones del pensamiento por medio de las cuales el sujeto puede apropiarse de los contenidos y del proceso que usó para ello; un conjunto de operaciones mentales cuyo objetivo es que el alumno integre la información adquirida básicamente a través de los sentidos, en una estructura de conocimiento que tenga sentido para él.

Las "habilidades cognitivas" referidas a la capacidad -o competencias, en su caso- adquiridas por el alumno refieren a una categorización que es definida por su "dominio".

Estos niveles de dominio se contestan en la medida de la respuesta a la pregunta: ¿Cuál es la diferencia entre los estudiantes justo al inicio de sus estudios y aquellos que están cerca de graduarse? (Sánchez, 2004). El dominio se organiza en tres niveles (Sánchez, 2004): inicial, medio y superior, de conformidad con la siguiente tabla, que especifica los criterios para cada uno (ver Imagen 4).

\begin{tabular}{|c|c|c|}
\hline \multirow{2}{*}{\multicolumn{3}{|c|}{$\begin{array}{c}\text { ¿Qué esperamos del estudiante } \\
\text { en los cursos iniciales? }\end{array}$}} \\
\hline & & \\
\hline $\begin{array}{l}\text { Criterio 1: } \\
\text { Exigencia mínima } \\
\text { Autoconocimiento } \\
\text { Autoevaluación } \\
\text { Autoobservación } \\
\text { Identificación en sí } \\
\text { mismo de los propios va } \\
\text { Demostración de una } \\
\text { postura propia }\end{array}$ & $\begin{array}{l}\text { Criterio 1: } \\
\text { Exigencia media } \\
\text { Aplicar } \\
\text { Analizar }\end{array}$ & $\begin{array}{l}\text { Criterio 1: } \\
\text { Exigencia máxima } \\
\text { Integrar }\end{array}$ \\
\hline $\begin{array}{l}\text { Criterio 2: } \\
\text { Contextos habituales } \\
\text { Aula } \\
\text { Biblioteca } \\
\text { Terreno } \\
\text { Educa } \\
\text { Otros }\end{array}$ & $\begin{array}{l}\text { Criterio 2: } \\
\text { Contextos diferentes } \\
\text { Laboratorio } \\
\text { Casos inventados } \\
\text { Problemas inventados } \\
\text { Simulaciones } \\
\text { Otros }\end{array}$ & $\begin{array}{l}\text { Criterio 2: } \\
\text { Contextos múltiples } \\
\text { y complejos } \\
\text { Prácticas } \\
\text { Situación profesional } \\
\text { Casos reales } \\
\text { Problemas reales } \\
\text { Otros }\end{array}$ \\
\hline $\begin{array}{l}\text { Criterio disciplina: } \\
\text { Conocimientos básicos, } \\
\text { generales, concretos } \\
\text { Fundamentos teóricos } \\
\text { de la profesión }\end{array}$ & $\begin{array}{l}\text { Criterio disciplina: } \\
\text { Ténicas y metodologías } \\
\text { de la profesión }\end{array}$ & $\begin{array}{l}\text { Criterio disciplina: } \\
\text { Conocimientos profundos, } \\
\text { específicos, abstractos }\end{array}$ \\
\hline $\begin{array}{l}\text { Criterio tarea: } \\
\text { Concreta o específica } \\
\text { Unidimensional } \\
\text { Poco profunda } \\
\text { Lenguaje técnico básico } \\
\text { Individual } \\
\text { Dependencia } \\
\text { Control e intervención } \\
\text { del profesor }\end{array}$ & & $\begin{array}{l}\text { Criterio tarea: } \\
\text { Compleja } \\
\text { Multidimensional } \\
\text { Muy profunda } \\
\text { Académico-investigación } \\
\text { Diseño y creación } \\
\text { Lenguaje técnico-técnico } \\
\text { Colectivo } \\
\text { Autonomía } \\
\text { Autogestión del aprendizaje }\end{array}$ \\
\hline
\end{tabular}

Imagen 4. Niveles de dominio. Fuente: Sánchez, 2009.

8 El concepto de "habilidades cognitivas" proviene del campo de la psicología cognitiva. Adaptado de la ppt de la Dra. Canny Bellido, Universidad de Puerto Rico Mayagüez. 


\section{Operacionalización de la tabla de seguimiento}

Para facilitar el diseño y la revisión de los programas de materias y que éstos respondan tanto a los objetivos de aprendizaje establecidos en el nuevo plan de estudios como a la relación que deben tener entre sí las materias de cada semestre, de éstas con otras del semestre anterior, así como la relación con las materias que le preceden en el siguiente semestre, se elaboró una tabla por semestre en la que se encuentran los siguientes datos:

Para dar seguimiento a los objetivos y contenidos de un programa de materia, se puede usar la tabla de la siguiente manera:

\begin{tabular}{|c|c|c|c|c|c|c|}
\hline Semestre & $\begin{array}{c}\text { Nombre de la } \\
\text { materia }\end{array}$ & Objetivo general & Nivel taxonómico & $\begin{array}{l}\text { Nivel de } \\
\text { dominio }\end{array}$ & Contenidos mínimos & Nivel taxonómico \\
\hline \multirow{4}{*}{$3^{\circ}$} & \multirow{4}{*}{$\begin{array}{l}\text { Teoría de la } \\
\text { arquitectura II }\end{array}$} & \multirow{4}{*}{$\begin{array}{l}\text { El estudiante comprenderá y } \\
\text { dominará los aspectos básicos } \\
\text { que conforman la Arquitectura, } \\
\text { especialmente aquellos referentes } \\
\text { a su ubicación en el contexto, su } \\
\text { funcionalidad y sus posibilidades } \\
\text { constructivas. }\end{array}$} & \multirow{4}{*}{$\begin{array}{l}\text { BAJO: CONOCER/ } \\
\text { COMPRENDER/ } \\
\text { APLICAR }\end{array}$} & \multirow{4}{*}{1} & Concepto del lugar. & Explicar \\
\hline & & & & & Aspectos fisicos y culturales de la Arquitectura. & Reconocer \\
\hline & & & & & La función en la Arquitectura. & Explicar \\
\hline & & & & & $\begin{array}{l}\text { Elementos que determinan el género } \\
\text { y la tipologia. }\end{array}$ & Reconocer \\
\hline \multirow{3}{*}{$3^{\circ}$} & \multirow{3}{*}{$\begin{array}{l}\text { Historia de la } \\
\text { arquitectura II }\end{array}$} & \multirow{3}{*}{$\begin{array}{l}\text { El estudiante analizará diversos } \\
\text { espacios habitables del periodo } \\
\text { comprendido en el curso, en } \\
\text { conexión con las ideas, la ciudad, } \\
\text { la naturaleza, el territorio y los } \\
\text { aparatos y sistemas artificiales } \\
\text { de producción resultantes de } \\
\text { la visión técnico-científica del } \\
\text { mundo y que influyeron en la vida } \\
\text { cotidiana de las poblaciones. }\end{array}$} & \multirow{3}{*}{ ALTO: ANALIZAR } & \multirow{3}{*}{2} & $\begin{array}{l}\text { Los espacios habitables en el Renacimiento, el } \\
\text { Barroco y la llustración. }\end{array}$ & Clasificar \\
\hline & & & & & $\begin{array}{l}\text { Los espacios habitables en los tiempos de la } \\
\text { Revolución Industrial. }\end{array}$ & Clasificar \\
\hline & & & & & Los espacios habitables en el Siglo xix. & Clasificar \\
\hline \multirow{5}{*}{$3^{\circ}$} & \multirow{5}{*}{$\begin{array}{l}\text { Taller de } \\
\text { maquetas }\end{array}$} & \multirow{5}{*}{$\begin{array}{l}\text { El alumno conocerá y se habilitará } \\
\text { en las técnicas de elaboración } \\
\text { de maquetas en diferentes } \\
\text { materiales para la representación } \\
\text { de proyectos arquitectónicos y el } \\
\text { ambiente que los rodea. }\end{array}$} & \multirow{5}{*}{ ALTO: ANALIZAR } & \multirow{5}{*}{3} & $\begin{array}{l}\text { Catálogo de materiales empleados en la } \\
\text { elaboración de maquetas. }\end{array}$ & Clasificar \\
\hline & & & & & $\begin{array}{l}\text { Técnicas de corte, ensamble y pegado de } \\
\text { diferentes materiales. }\end{array}$ & Aplicar \\
\hline & & & & & $\begin{array}{l}\text { Realidad: abstracciones que sustituyen la } \\
\text { realidad, escala, colores, texturas, elementos } \\
\text { ambientales. }\end{array}$ & Interpretar \\
\hline & & & & & Elaboración de paletas de materiales y colores. & Clasificar \\
\hline & & & & & $\begin{array}{l}\text { Composición plástica en las representaciones } \\
\text { de proyectos arquitectónicos. }\end{array}$ & Modelar \\
\hline
\end{tabular}

Columnas

Aspectos a tomar en cuenta en el diseño y revisión de programas de materias para su implementación.

1. Tener a la mano la tabla correspondiente al semestre en la que se encuentra la materia que impartirá, así como las tablas del semestre anterior y posterior a éste; por ejemplo: si la materia es Historia de la Arquitectura II, debo tener la tabla del tercer semestre, ya que en éste es donde se imparte esa materia, además es necesario tener a la mano las tablas del segundo y cuarto semestres.

2. Identificar la fila que corresponde a la materia de la cual se diseñará o revisará el programa.

3. Utilizar el formato institucional para programas de materias, éste se puede descargar del Sistema de Gestión de la Calidad (SGC) en la página web de la UAA (https://sgc.uaa.mx/documentosF/index.php\#ad10).

4. Llenar los datos de identificación.

5. Escribir la descripción general de la materia, retomándola del plan de estudios vigente.

6. En el mismo plan de estudio, o dirigiéndose a la tabla, anotar el objetivo general de la materia; éste, al igual que la descripción del punto anterior, no pueden ser modificados porque ya están autorizados. Si fuera necesario, se pueden hacer recomendaciones a la academia correspondiente para que se tomen en cuenta para la próxima revisión del plan de estudios.

7. El nivel taxonómico corresponde al objetivo; en la tabla debe corres- 
ponder con las características de la materia.

8. El nivel de dominio ayudará a implementar estrategias de aprendizaje adecuadas para el semestre en el que se encuentran los estudiantes.

9. Los contenidos mínimos, también obtenidos del plan de estudios vigente, se retoman para diseñar las diferentes unidades de aprendizaje; no será necesario que cada contenido conforme una unidad, podrían ser agrupados, dependiendo de las necesidades de cada materia.

10. El nivel taxonómico de cada contenido recomienda un verbo que puede ser utilizado para la redacción de objetivos particulares de esas unidades (ver Imagen 3).

11. El programa debe completarse con la metodología, recursos didácticos, evaluación y bibliografía. Para la evaluación también se toma en cuenta la columna del nivel de dominio de competencias, ya que éste considera conocimientos, habilidades y actitudes (ver Imagen 4).

\section{Conclusión}

Esta metodología pretende, en un modelo de universidad departamental, coadyuvar en la planeación de la enseñanza y aprendizaje de un programa académico, con los trabajos de las distintas academias que lo soportan, a partir de una adecuada implementación y seguimiento de un plan de estudios para la calidad en el logro de sus objetivos.

\section{Fuentes de consulta}

Anderson, L.W., Krathwohl, D.R., Bloom, B.S. (2001). Una taxonomía para el aprendizaje, enseñanza y evaluación: una revisión de la taxonomía de Bloom de objetivos educativos. Boston, MA: Allyn \& Bacon, Pearson Educación grupo.

Bloom, B. et al. (1971). Taxonomía de los objetivos de la educación: la clasificación de las metas educacionales: manuales I y II (traducción de Marcelo Pérez Rivas; prólogo del profesor Antonio F. Salonía). Buenos Aires: Centro Regional de Ayuda Técnica: Agencia para el Desarrollo Internacional (A.I.D).

Pérez Torres, I. (2006). WebQuests: Habilidades cognitivas. En Presentación Jornadas Web Quest. Barcelona. Consultado en julio 2013 en http://www.slideshare.net/juanw/jornadas-wq-13399082.

Sánchez Doberti, T. (Dir.). (2009). Orientaciones para la renovación curricular, Etapa 2, Chile, Dirección General de Docencia, Universidad Católica de Tamuco. Consultado en julio 2013 en http://repositoriodigital.uct.cl:8080/xmlui/bitstream/handle/123456789/516/Renovacion_curricular_guia2.pdf?sequence=5.

Universidad Autónoma de Aguascalientes. (2007). Modelo Educativo Institucional. Correo Universitario, sexta época, núm.16, publicado el 15 de marzo de 2007. México: UAA.

Universidad Autónoma de Aguascalientes. (2012). Plan de Estudios de la Licenciatura en Arquitectura 2012. Departamento de Diseño del Hábitat, cCDC, UAA. 